\title{
SOME OBSERVATIONS OF SHORT-PERIOD RADIO
} FADING

\author{
By T. Parkinson
}

\section{ABSTRACT}

The data presented are the product of an investigation started at the beginning of 1928 with the object of studying the short-period fading of radio broadcast transmissions. Particular attention was paid to those intensity changes which take place during periods ranging from a few seconds to several minutes. Various antenna combinations were used in making simultaneous records in order to separate the effects of various causes of fading.

It was concluded that, in addition to the more commonly accepted causes, there may be a number of indirect rays with varying paths and with rotating planes of polarization which give rise to the variations in intensity of the received signal. Some of the conclusions are consistent with those of other workers in the field, but new explanations are necessary for some of the data.

\section{CONTENTS}

I. Introduction

II. Method and apparatus

III. Results___._. 1059

1. Simultaneous coil and vertical single-wire antenna records_._- 1059

2. Simultaneous fading records with two coil antennas at maximum and minimum positions, respectively _........... 1064

3. Simultaneous fading records made with barrage antenna and with vertical single-wire or coil antenna _._._._._._._._. 1068

4. Short-period fading . _.

IV. Conclusions _... 1072

V. Acknowledgments... 1073

VI. Appendix _... 1073

1. Summary of conclusions of previous investigations.

\section{INTRODUCTION}

The data presented in this paper are the product of an investigation started by the Bureau of Standards at the beginning of 1928 with the object of studying the short-period fading of radio broadcast transmissions. This study was directed particularly to those intensity changes in the receiving antenna which range, in period, from a few seconds to several minutes between peaks. These variations have been variously ascribed by previous investigators to one or more of the following causes: (1) Intensity changes undergone by the indirect or atmospheric ray, namely, that portion of the radiation which, at night especially, travels to the upper atmosphere and is returned to earth at the receiving antenna by refraction; (2) inter- 
ference, due to varying phase relationships of two or more indirect rays or of an indirect ray and the ground ray, which follows a direct path along the surface of the earth from transmitting to receiving antenna; (3) direction shifts, this expression being used to connote deviations of the indirect ray from the great circle plane passing through transmitting and receiving points; (4) changes in the angle of incidence (that is, in the vertical plane of arrival of the indirect ray); (5) rotation of the plane of polarization of the atmospheric ray, this rotation being considered as a continuous process rather than in the optical sense of a definite displacement of the plane at completion of the process. The aim of the present investigation was to separate the effects due to the above-mentioned causes with a view to determining to what extent each might be a factor in fading.

\section{METHOD AND APPARATUS}

The method consisted in selecting a particular broadcasting station and making simultaneous graphic records of its carrier wave as received by different types of antennas attached to duplicate receiving sets. The antennas used were (1) a vertical single wire, $15 \mathrm{~m}$ high, with a $2 \mathrm{~m}$ horizontal lead-in; (2) a vertical coil having its turns in a great circle plane common to transmitting and receiving points, which coil will hereafter be referred to as "at maximum," or "in the maximum position"; (3) a vertical coil perpendicular to the great circle plane and hereafter to be designated as "at minimum," or "in the minimum position"; (4) a so-called barrage antenna, consisting of the singlewire antenna so coupled to a coil antenna in the maximum position as to introduce an out-of-phase current and thus neutralize the effect of the ground ray as well as of the vertical component of the indirect ray. The coil antennas were so balanced by a center-tap arrangement as to eliminate any appreciable "antenna effect." For all tests the various antennas were located from 3 to $5 \mathrm{~m}$ apart. These distances were sufficiently small so that records obtained simultaneously with similar antennas were alike, and were sufficiently large to prevent interaction between the separate receiving circuits. All observations were made at the Bureau of Standards field station located at Kensington, Md., $8 \mathrm{~km}$ north of the bureau and in a position practically free from "man-made" interference and distorting influences.

Other apparatus employed was essentially that described by G. W. Pickard ${ }^{1}$ in 1923 and used later in the cooperative investigation conducted by the Bureau of Standards in $1925 .^{2}$ The receiving sets were superheterodynes of the same make and were capable of adjustment to practically the same amplification. The driving motors of

\footnotetext{
1 G. W. Pickard, Short-period Variations in Radio Reception, Proc. Inst. Radio Eng., 12, pp. 119-158; April, 1924.

2 J. H. Dellinger, C. B. Jollifie, and T. Parkinson, Cooperative Measurements of Radio Fading in 1925, B. S. Sci. Paper No. 561, pp. 423-425.
} 
the Shaw manual recorders were automatically synchronized every second, thus making it possible to compare two records by superposition.

Observations were concentrated largely upon two stations, but less-frequent records were made on transmissions from other stations involving frequencies of 550 to $1,480 \mathrm{kc}$ and direct-transmission paths of 13 to $1,500 \mathrm{~km}$. The greater number of records were made upon WJZ, Boundbrook, N. J., and upon WBAL, Baltimore, Md., because of the regularity with which each could be received, day or night, and because of what appeared to be characteristic features. Many of the observations started during daylight when there was no appreciable indication of an indirect ray and when, consequently, the most accurate bearings could be secured with the coil antennas. At this time, also, the two receiving systems could be so adjusted that the ground wave produced the same deflection on the recorder galvanometers of the two receiving systems. When records were started after dark no attempt was made to have them on an accurately comparable intensity basis, the chief aim being to study the simultaneous changes on two records rather than their exact magnitudes.

The arrangement of the pairs of simultaneous records in the illustrations will be found somewhat inconsistent, since the character of the records made it easier to compare them by placing the one made with a given type of antenna sometimes above, sometimes below the record made with another type.

\section{RESULTS}

\section{SIMULTANEOUS COIL AND VERTICAL SINGLE-WIRE ANTENNA RECORDS}

The first method of analysis in the present investigation was by means of simultaneous fading records made with coil antenna in maximum position and with vertical single-wire antenna, respectively. Assuming both ground and reflected rays to be in the great circle plane which passes through transmitting and receiving points, certain effects were anticipated. The ground ray presumably remains normally polarized at night as during daylight; therefore, so long as there is a normally polarized component of the indirect ray any changes in the path length of the latter should result in changing phase relations between the two rays, and, consequently, in similar and simultaneous intensity variations in the two types of antenna. Since the total electric vector in the vertical plane is effective on the coil antenna, the signal strength in it should range between the sum and the difference of the two fields produced by direct and indirect rays, respectively. On the vertical single-wire antenna similar variations should be of less absolute magnitude, since only the vertical component of the electric vector is effective and this component decreases with 
the angle of incidence. With either antenna the night intensity, for nonlocal stations at least, will sometimes exceed, sometimes fall below the relatively constant daylight level, and similar variations should occur simultaneously on both records.

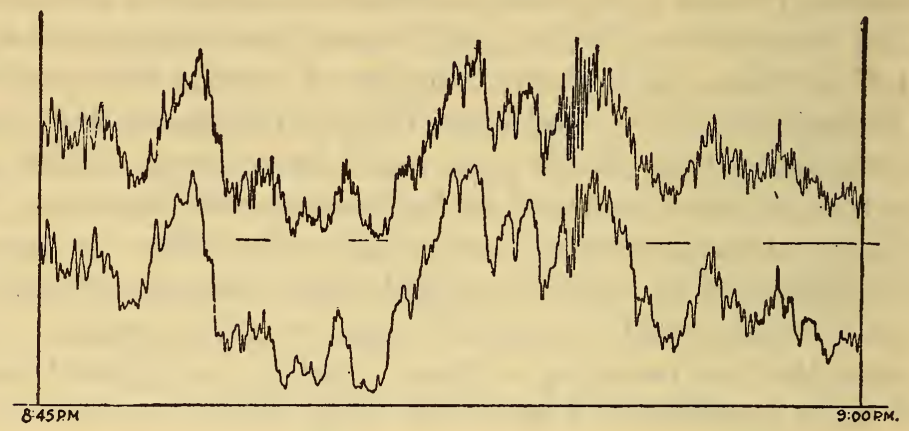

Figure 1.-WIOD $(1,210 \mathrm{kc})$, Miami Beach, Fla., rereived at Kensington, $M d$., 1,500 km, March 15, 1928

Upper record with vertical single-wire antenna; lower record, coil antenna at maximum.

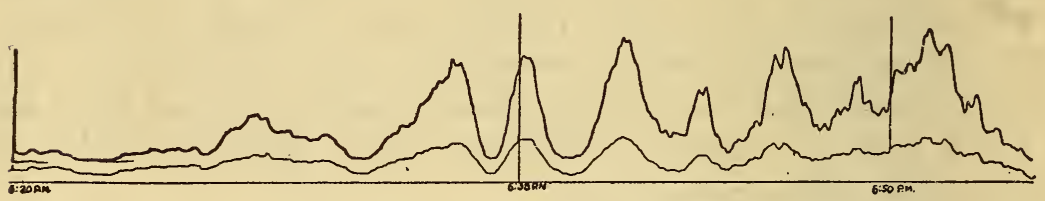

Figure 2.-WJZ $(660 k c)$, Boundbrook, N. J., received at Kensington, Md., $300 \mathrm{~km}$, May 15, 1928

Upper record, coil antenna at maximum; lower record, vertical single-wire antenna.

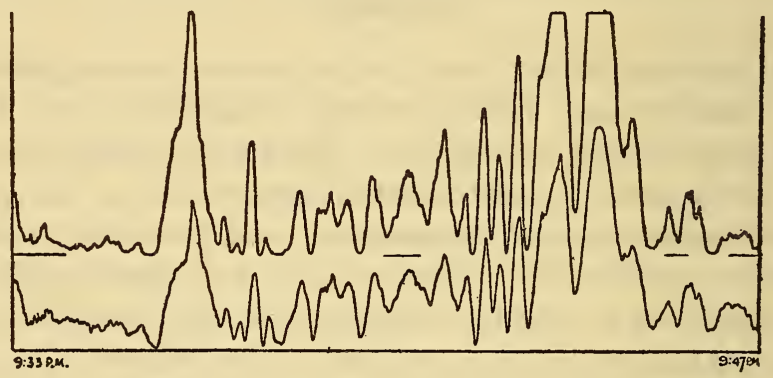

Figure 3.-WRVA $(1,180 \mathrm{kc})$, Richmond, Va., received at Kensington, Md., $155 \mathrm{~km}$, June 1, 1928

Upper record, coil antenna at maximum; lower record, vertical singlewire antenna.

Turning now to the data and examining some 50 records involving transmission frequencies of 660 to $1,480 \mathrm{kc}$ and distances of 13 to $1,500 \mathrm{~km}$, what do we find? For stations more than about $150 \mathrm{~km}$ distant from the receiving antennas the results were practically as anticipated, as the typical graphic records of Figures 1 to 3 indicate. 
There is a slight shifting of phase at one point in the WIOD record shown in Figure 1 which may be real or may have been caused by changing speed of one recording drum at a time when there was difficulty with the synchronization of the two drums. Conditions prevented making another record on this station. With this single excep-

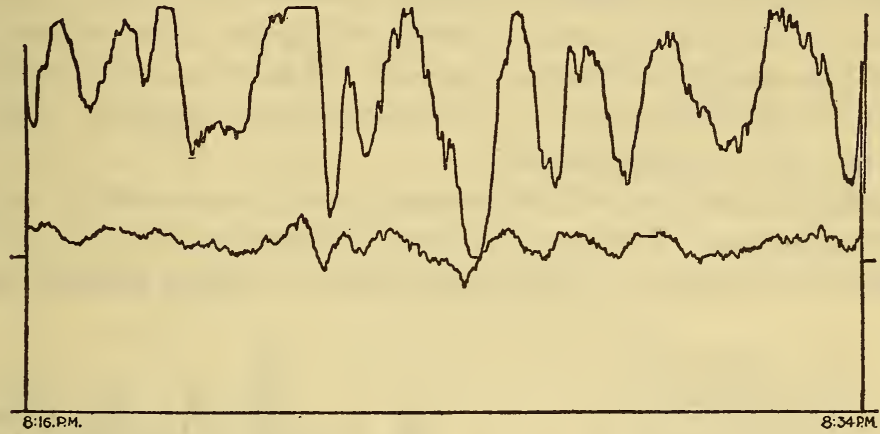

Figure 4.-WBAL $(1,050 \mathrm{kc})$, Baltimore, Md., received at Kensington, $M d$., $50 \mathrm{~km}$, May 9, 1928

Upper record, coil antenna at maximum; lower record, vertical single-wire antenna.

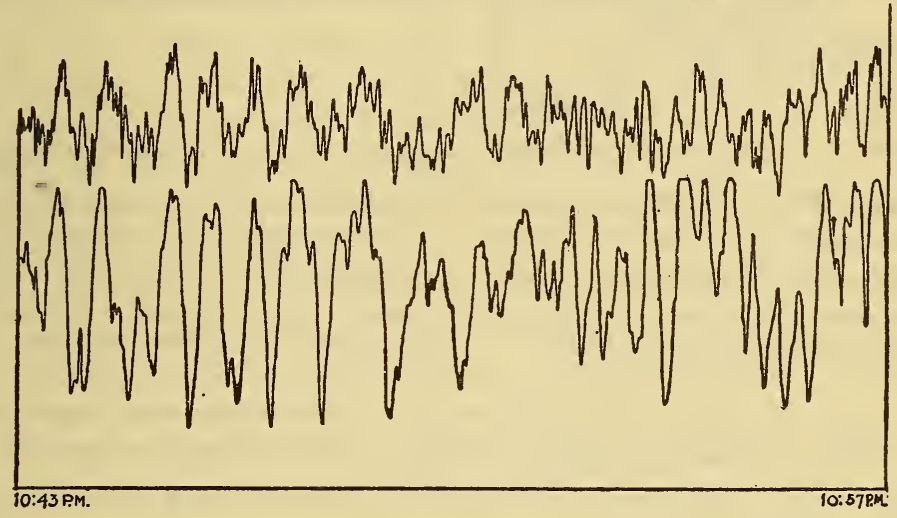

Figure 5.-WTFF (1,480 kc), Mount Vernon Hills, Va., received at Kensington, Md., $31 \mathrm{~km}$, March 19, 1928

Upper record, vertical single-wire antenna; lower record, coil antenna at maximum.

tion the intensity changes are in phase on the two antennas. An anomaly appears, however, in the records for the nearer group of stations (13 to $53 \mathrm{~km}$ distant) in that the intensity changes are at times as much as $180^{\circ}$ out of phase on the two records. This holds true for variations of a few seconds duration as well as for those having periods of a minute or two. Figures 4 to 7 show typical illustrations. Here we have the same effect that Appleton and Ratcliffe ${ }^{3}$ found in

${ }^{3}$ E. V. Appleton and J. A. Ratcliffe, On the Nature of Wireless Signal Variations, Proc. Royal Soc., 115A, pp. 291-317; June, 1927. See especially pp. 311-312 and fig. 9. 
simultaneous records made with vertical single-wire antenna and with the ground ray suppressed in a single-turn loop placed in the maximum position. They accounted for the recorded differences by an interference between indirect and ground rays which was effective in the vertical antenna but was eliminated from the loop. This explanation is not applicable to the present data, since with the ground ray effective in both antennas there is still a lack of synchronism in the intensity changes of the two records. Appleton and Ratcliffe, on the other hand, found the two records varying together when the ground ray was not suppressed. ${ }^{4}$

A possible explanation of this anomaly may be reached by the process of elimination. Making the usual assumption that the indirect ray remains in the plane of the great circle connecting transmitter and

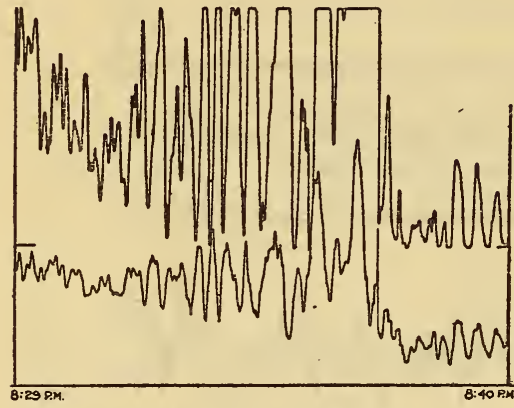

Figure 6.-WTFF $(1,480 \mathrm{kc})$, Mount

Vernon Hills, Va., received at Kensington, Md., $31 \mathrm{~km}$, May 17, 1928

Upper record, coil antenna at maximum; lower record, vertical single-wire antenna.

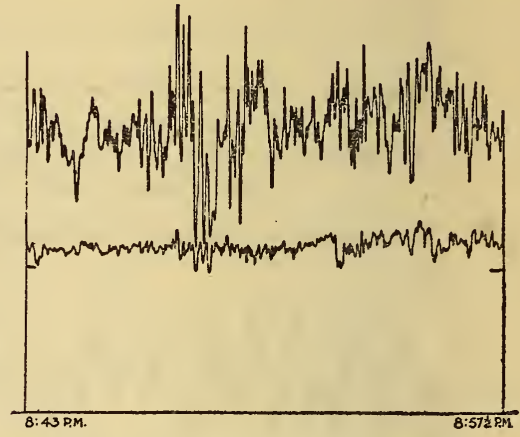

Figure 7.-WMAL (1,240 $k c)$, Washington, D. C., received at Kensington, Md., $13 \mathrm{~km}$, May 7, 1928

Upper record, coil antenna at maximum; lower record, vertical single-wire antenna.

receiver, the combined effect of ground and downcoming rays should be to cause similar changes in both vertical single-wire and coil antennas. The only exception would be when the atmospheric ray arrived with zero angle of incidence, in which case only the ground ray should be effective in the vertical antenna and the intensity should remain at the daylight level, while in the coil antenna the combined effect of the two rays should continue to produce fading changes. This explanation does not fit the data; it is a change of phase relationship between the two records, not a cessation of fading in one, that that is to be accounted for. Rotation of the plane of polarization of the incident ray also should produce like effects in the two receiving systems; therefore, by itself, this offers no solution.

The answer is presumably associated, directly or indirectly, with the nearness of transmitting and receiving stations. For the distances

- Reference 3, p. 300, figs. 4 and 5. 
concerned, 13 to $53 \mathrm{~km}$, it is perfectly possible to have direction shifts of the incident ray as great as $90^{\circ}$, due to irregularities in the refracting or reflecting upper atmosphere. This could account for changes on the vertical antenna not occurring on the coil antenna, but not for the considerable time lag between similar changes produced in the two receiving systems. If, however, there should be a lateral direction shift combined with a rotation of the plane of polarization for the downcoming ray, we should expect just such differences in the pairs of records as appear. Suppose, for purposes of illustration, that the reflected ray is arriving with constant intensity and normally polarized. If, now, the lateral angle of arrival shifts until it becomes $90^{\circ}$, the signal intensity in the vertical antenna will remain constant while that in the coil antenna will decrease to a minimum; in fact, to zero, if there should be no ground wave present.

If, next, the plane of polarization rotates, the vertical component of the electric field will decrease until, with the electric field horizontal, the ray ceases to produce a current in the vertical antenna. Simultaneously, the component of the magnetic field in the vertical plane will have increased from zero to a maximum, thereby producing maximum current in the coil antenna for the given angle of incidence. Thus we have similar fading characteristics $180^{\circ}$ out of phase in the two antennas. If the direction of arrival of the incident ray gradually returns to the plane of the maximum coil antenna and the rotation of the plane of polarization continues, whether at regular or irregular speed, the phase difference between the two records will gradually decrease until it disappears. With the direction shifts more or less erratic we should have just the sort of changing phase relations shown on the records of Figures 4 to 7 ; and if rotation ceased, we should again find the two records running in phase, as in the early part of Figure 5 or the latter part of Figure 6.

The question whether the direction shifts necessary to the above explanation actually exist requires proof. Bearings on these stations were taken at night by means of a coil antenna and revealed apparent direction shifts which were considerable. An actual test, however, can only be made with an antenna of the Adcock type ${ }^{5}$ if true directions of arrival of the reflected ray are to be found. Such a test awaits the construction of further apparatus.

Interference between the indirect ray and the ground ray is evidenced on many of the records made with the coil antenna in maximum position, and to a lesser degree by those made with the vertical singlewire antenna. Wherever the intensity of one of these records falls

5 Improvement in means for determining the direction of a distant source of electromagnetic radiation. British Patent 130490, 1919. For actual applications, see reference 14 in appendix. 
below the daylight level the indirect ray must be to some extent out of phase with the waves arriving along the surface of the earth. Figures 4 to 7 give clear indications of this. Note particularly that even for stations as near as $13 \mathrm{~km}$ from the observing point, as shown by Figure 7, the intensity of the downcoming ray is sometimes nearly as great as that of the ground wave, so that when the two are opposite in phase the intensity of the ground ray is largely neutralized and the record drops almost to zero. Presumably in these same records the peaks are due to the addition of the reception by direct and indirect paths. It will later be shown, however, that peaks may be due to other causes, whether the ground wave be present or not.

\section{SIMULTANEOUS FADING RECORDS WITH TWO COIL ANTENNAS AT MAXIMUM AND MINIMUM POSITIONS, RESPECTIVELY}

The second method of analysis was by simultaneous fading records of the same transmission as received by two coil antennas, one in the maximum and the other in the minimum position. The purpose of

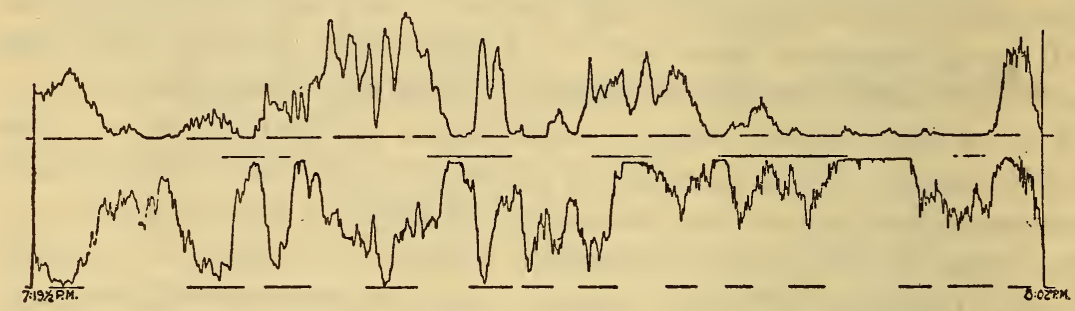

FIGURe 8.-WJZ (660 kc), Boundbrook, N. J., received at Kensington, Md., $300 \mathrm{~km}$, April 2, 1928

Upper record, coil antenna at maximum; lower record, coil antenna at minimum position.

this arrangement was to secure some data as to fading caused by rotation of the plane of polarization of the indirect ray, for such a phenomenon, if present, should cause an increase of intensity in either coil antenna to be accompanied by a decrease in the other. So far as possible the minimum bearing was found for the various stations during daylight when there was no evidence of an indirect ray, and these bearings checked satisfactorily on different days. For the more distant stations, however, no ground wave could be received in daylight, and it was necessary to trust to approximate bearings, which were sufficient for the project in hand.

The data secured offers considerable striking evidence of the abovementioned phenomenon. Figure 8 is a typical section cut from a pair of simultaneous records. Here it is possible to see at a glance that the two are practically $180^{\circ}$ out of phase most of the time. The minima do not always fall to zero as would be expected with the ground wave practically negligible, as it is in this case, and it, therefore, becomes necessary to postulate more than one indirect ray. 
It may be that in this record we have the effect of two rays rotating at different speeds so that their minima do not synchronize most of the time. Interference between the two is also probably present, but the effect can not be isolated in the record. Figure 9 is another record made on reception from the same station as Figure 8 and is typical of what occurs during the sunset period. Up until a half hour after sunset the weak ray from the upper atmosphere appears to remain normally polarized and so produces intensity changes only in the coil at maximum position, possibly through a combination of interference effects and varying absorption. Then gradually evidence appears that an indirect ray somewhat abnormally polarized is producing a current in the minimum coil. With no further rotation of the plane of polarization any variations in the intensity of the indirect ray may now result in parallel changes suck as appear in the first large intensity peak at 7.12. Here, due probably to the combined effects of very abnormal polarization and of small angle of incidence

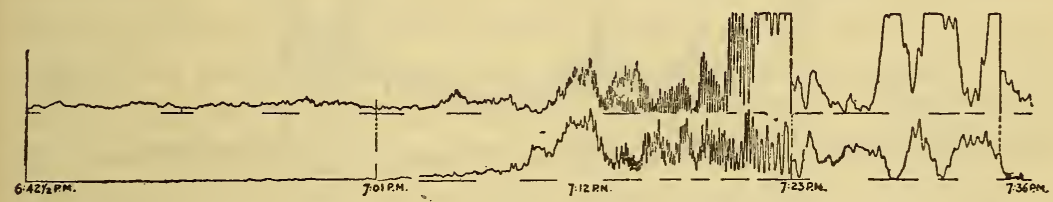

Figure 9.-WJZ (660 kc), Boundbrook, N. J., received at Kensington, Md., $300 \mathrm{~km}$, April 5, 1928

Local sunset at 6.36. Upper record, coil antenna at maximum; lower record, coil antenna at minimum.

accompanying increased height of the ionized layer, the intensity in the coil at minimum is practically the same as in that at maximum. From this peak on, nearly to the end of the record, the average trend of the maximum coil antenna record is $180^{\circ}$ out of phase with the minimum, indicating that the rotation of the plane of polarization of the indirect ray has gotten under way. Starting at about 7.04 p. m. on this same record there is a superposed rapid variation having a period of from 3 to 30 seconds. This characteristic is also exactly out of phase on the two records and so suggestive of a second and relatively much weaker ray with plane of polarization rotating rapidly. Assuming the polarization to be plane, a complete rotation would have to occur in six seconds to produce the most rapid of these changes.

While these data and much more seemed to be consistent with rotation of the plane of polarization in the indirect ray, there were times when the phase difference between the two records varied widely, sometimes becoming almost zero, as shown in Figures 10 to 12 . The ground ray could in no way be held responsible, for its effect had been eliminated by the coil antenna in minimum position, and yet variations in one antenna led or lagged behind similar variations in the 
other. In a recent article Eckersley ${ }^{6}$, reports that records of highfrequency transmissions received simultaneously with vertical and

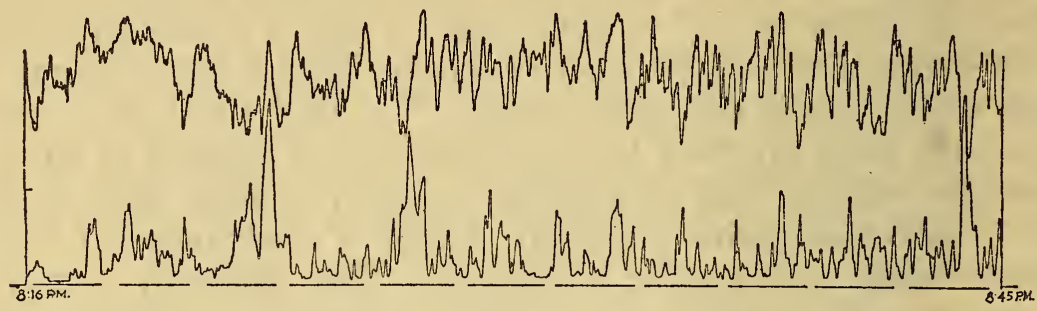

Frgure 10.-WBAL $(1,050 \mathrm{kc})$, Baltimore, $M d$., received at Kensington, $M d$., $50 \mathrm{~km}$, April 17, 1928

Upper record, coil antenna at maximum; lower record, coil antenna at minimum.

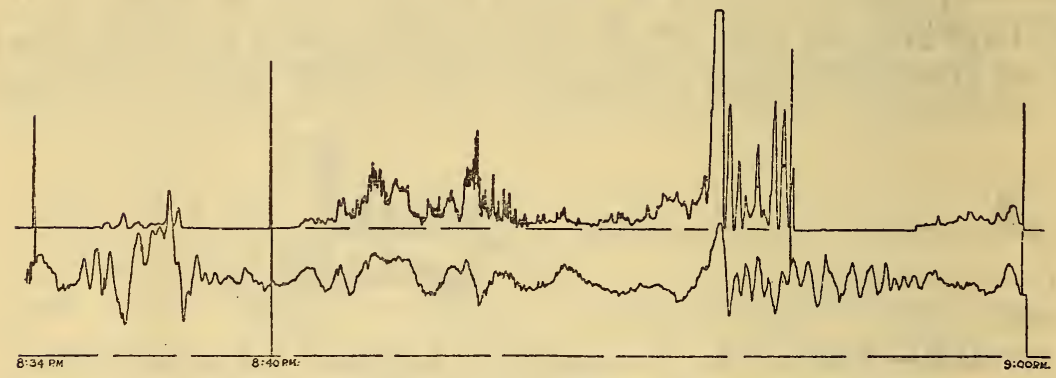

Figdre 11.-WMAL (1,240 kc), Washington, D. C., received at Kensington, Md., $13 \mathrm{~km}$, June 5, 1928

Upper record, coil antenna at minimum; lower record, coil antenna at maximum.

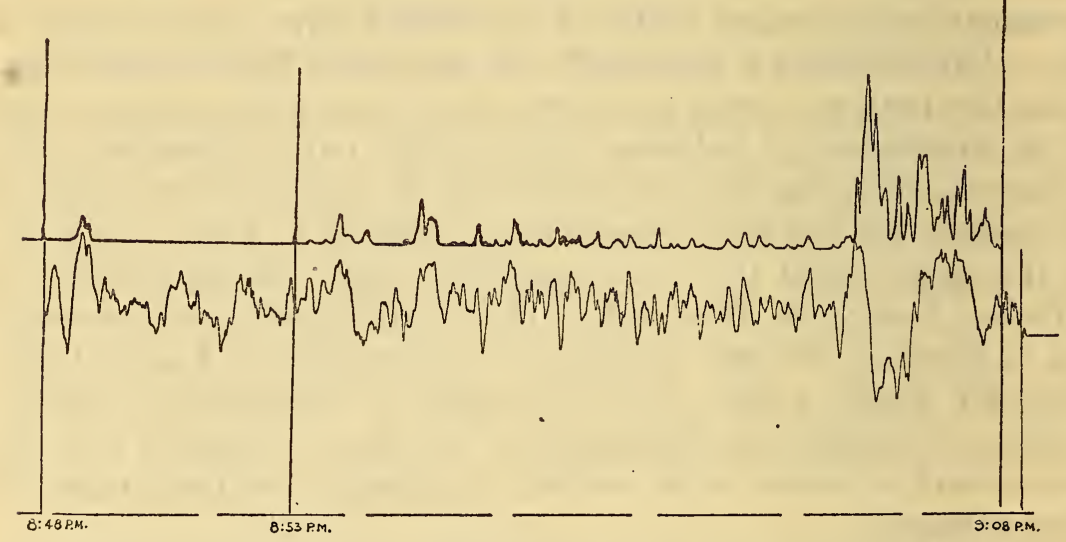

Figure 12.-WMAL (1,240 kc), Washington, D. C., received at Kensington, Md., $13 \mathrm{~km}$, June 6, 1928

Upper record, coil antenna at minimum; lower record, coil antenna at maximum.

horizontal antennas are opposite in phase. It is interesting to note that the illustration given in that paper, however, shows clearly the 
same sort of phase shifts between the two records as have appeared in the data of the present paper. To account for this phenomenon by an interference effect, at least two elliptically polarized indirect rays would be necessary, with their vertically polarized components undergoing changes in phase relationship similar to but lagging or leading those of the horizontally polarized components.

A more plausible explanation is that suggested for similar out-ofphase relations which occurred in the records made simultaneously on coil and vertical-wire antennas. If an indirect ray with rotating plane of polarization were arriving with an angle of incidence of nearly $90^{\circ}$ and the plane of its arrival should gradually shift laterally from $0^{\circ}$ to $45^{\circ}$ with the maximum position of the coil antenna, the records made simultaneously by coil antennas in maximum and minimum positions would gradually shift from the $180^{\circ}$ out-of-phase relationship to a nearly-in-phase relationship. If the angle of incidence were considerably less than $90^{\circ}$, similar changes in phase relationships would take place as the direction of arrival shifted to the $45^{\circ}$ position, except that the two records would never come completely into phase. As the angle of incidence decreased still further, the two records would be more and more out of phase. If the indirect rays always arrive by means of a single reflection, the angle of incidence at the receiving end would increase with the distance between transmitting and receiving points, and a direction shift of given angle would produce a greater phase shift in the pair of fading records for a distant than in the pair for a nearer station because the incident ray for the former is more nearly horizontal. On the other hand, it should be possible for the ray from a nearer station to arrive at larger angles with the plane of the coil antenna in maximum position, since lateral radiation from the distant station would have to go to improbable distances to be reflected back at large angles. Very distant transmissions, furthermore, would presumably be received almost entirely in the great circle plane passing through transmitting and receiving stations. In the case of possible multiple reflections of the same ray or of an inverted $U$-shaped path the situation would be altogether different. Further measurements of the apparent effective height of the ionized region according to the method of Appleton and Ratcliffe, providing a sufficient variety of distances be covered, may give some useful information, for the method should prove most accurate and consistent for the distant stations if there are actual lateral reflections present.

As a partial check of the possible shifts in the direction of arrival of the indirect ray, observations were made simultaneously with three receiving systems utilizing, respectively, a coil antenna in maximum position, a second in the minimum position, and a third in a vertical plane at an angle of $45^{\circ}$ with the other two. Comparisons 
were made vocally by the three observers as changes occurred in the indicating galvanometers, since the third recorder for securing graphic records was lacking. Observing was done at a time when the phase relation between changes in the perpendicular coils was varying. The triple observations showed that for considerable periods the variations in the $45^{\circ}$ coil were more nearly in phase with those in the coil at maximum, at times more nearly in phase with changes in the coil at minimum position, and for other periods considerably out of phase with both. Such effects do not prove direction shifts

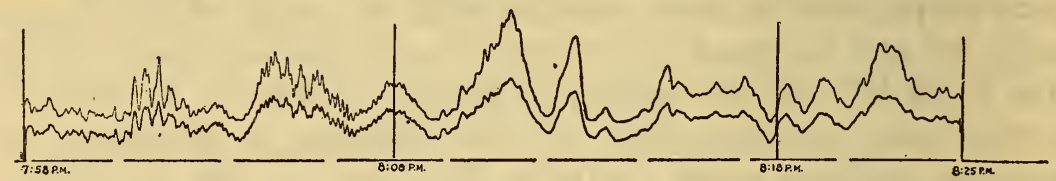

Figure 13.-WJZ $(660 k c)$, Boundbrook, N. J., received at Kensington, Md., $300 \mathrm{~km}$, June 6, 1928

Both records made on coils at maximum.

to exist but are similar to what would be expected if such shifts were real.

Figure 13 is typical of records taken from time to time to make sure that differences in simultaneous records were not due to the separation between receiving antennas. In all of these records made with similar antenna systems the simultaneous records showed that every variation, small and large, was in phase on the two records.

3. SIMULTANEOUS FADING RECORDS MADE WITH BARRAGE ANTENNA AND WITH VERTICAL SINGLE-WIRE OR COIL ANTENNA

Using the barrage combination of antennas to eliminate effects due to the ground wave, and the coil antenna in the maximum position

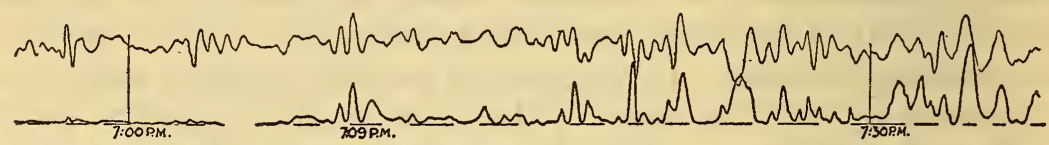

Figdre 14.-WBAL $(1,050 \mathrm{kc})$, Baltimore, $M d$., received at Kensington, $M d$., $50 \mathrm{~km}$, April 27, 1928

Local sunset at 6.58. Upper record, coil antenna at maximum; lower record, barrage minimum.

to receive both ground and indirect rays, it was hoped that differences in records would indicate the amount of fading due to interference between these two rays. With the indirect ray remaining in the plane of the great circle connecting the two stations, changes other than those due to interference should have similar effects on the two receiving systems, though the magnitude of changes should differ.

Figures 14 and 15 show typical pairs of records. The earlier portion of the records of Figure 14 occurs during sunset before the angle of 
incidence of the indirect ray has decreased sufficiently to produce more than a feeble horizontal component of the electric field. Consequently the intensity changes are quite feeble at first but increase as the ionized layer moves upward. During the first half of the records the intensity variations are in phase in the two antenna systems, indicating that these changes are produced farther back in the transmission path. The later portions of the records, however, show some

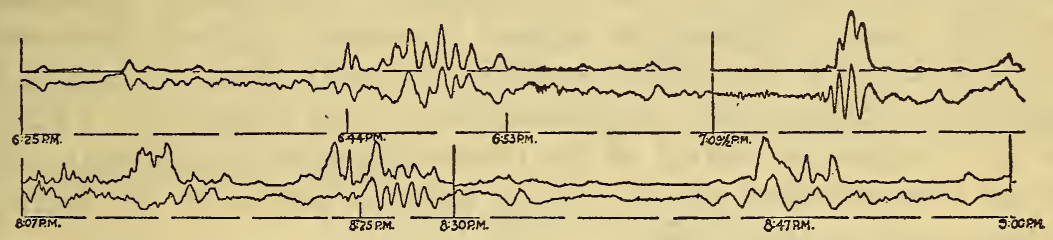

Figure 15.-WBAL $(1,050 \mathrm{kc})$, Baltimore, $M d$., received at Kensington, $M d$., $50 \mathrm{~km}$, April 26, 1928

Local sunset at 6.57. Upper record, barrage minimum; lower record, coil antenna at maximum.

changes in one antenna to be lagging similar changes in the other. Figure 15 shows similar lagging effects at approximately 6.44 to $6.53,8.25$ to 8.30 , and $8.47 \mathrm{p}$. m. At other times this pair of records is usually in phase or $180^{\circ}$ out. The latter condition is well explained by the fact that as the horizontal electrical component of the indirect ray increases, as shown by the barrage record, the vertical component presumably increases also and, being out of phase with the ground ray, whether partially or completely at these particular

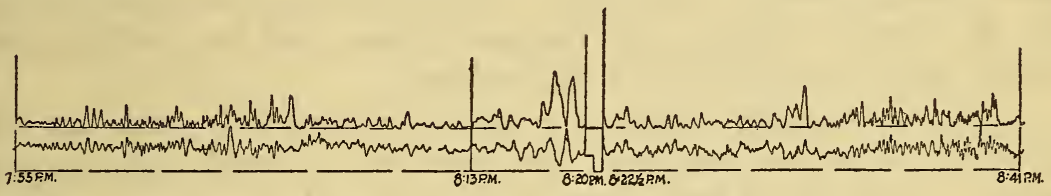

FIGURe 16.-WBAL (1,050 kc), Baltimore, $M d$., received at Kensington, $M d$., $50 \mathrm{~km}$, July 6, 1928

Upper record, barrage minimum; lower record, vertical single-wire antenna.

times, causes a reduction of intensity in the coil antenna set at maximum position.

Figure 16 shows records made by a method similar to that last described except that a vertical single-wire antenna was substituted for the coil in maximum position. This method was used by Appleton and Ratcliffe. ${ }^{7}$ Though the fading is more rapid in Figure 16, the phase relations between the records are similar to those of Figures 14 and 15, with less positive indications of the lagging effect because of the rapidity of variations. This effect does appear on a record ${ }^{7}$ 
shown by Appleton and Ratcliffe, however. The explanation given by them that the difference in records is due to the interference between indirect and ground rays which can affect only one of the two receiving systems is a possible one for all of these pairs of records. Two facts, however, cast some question upon this as a complete solution: (1) It is doubtful if interference can account for the slight phase displacements in similar portions of the two records, as it may well account for the $180^{\circ}$ out-of-phase relationships; (2) similar varying phase relationships appear in records already discussed, those made with the vertical antenna and with the coil antenna in maximum position with no elimination of the ground ray. Shifts in the direction of arrival of the down-coming ray would make the latter more effective on the vertical antenna portion of the barrage system than on either coil.

If rotating plane of polarization be combined with a direction shift in this ray, the phase relationships appearing in the pairs of simulta-

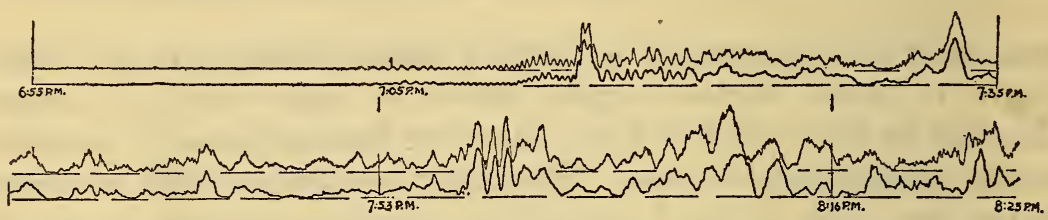

Figure 17.-WBAL (1,050 kc), Baltimore, $M d$., received at Kensigton, $M d$., $50 \mathrm{~km}$, March 30, 1928

Local sunset at 6.30. Upper record, coil antenna at minimum; lower record, barrage minimum.

neous records become explicable. Three simultaneous records utilizing the vertical antenna, the barrage system, and the coil antenna should throw further light on the problem.

Again, the barrage system was used simultaneously with the coil antenna in minimum position. This provided a method of eliminating results of the ground wave from both coils so that remaining effects should be due to indirect rays only and to the angles which the coils made with that indirect reception. The effect of the entire vertical electric component of this ray should, of course, be eliminated in the barrage system. Figure 17 shows the type of record secured on transmission during the sunset period from a station only $53 \mathrm{~km}$ distant. The changes, both large and small, are very nearly of the same magnitude, and the larger variations are usually in phase on the two records. The phase relationships of the smaller periodic variations change considerably but are most often $180^{\circ}$ out. In other words, this pair of records has much the same characteristics as appeared on the WJZ records, made with coils in maximum and minimum positions, in which the effect of the ground wave was practically absent because of great attenuation due to distance. There is in the early part of the records a superposed periodic varia- 
tion suggestive of an indirect ray of small intensity with rotating plane of polarization and some shift in the lateral angle of arrival to cause changing phase relationships in the two fading records. The major variations would appear to be caused by a much stronger indirect ray of varying intensity with its plane of polarization unchanging at first but later rotating slowly, thus causing changes in one record to lag behind similar changes in the other. This rotation effect was not found in the records made by Appleton and Ratcliffe ${ }^{8}$ with similar antennas. The intensity variations shown by their simultaneous records were so largely in phase that most of the fading could be explained by intensity variations occurring at some distance from the receiving antennas.

\section{SHORT-PERIOD FADING}

The rapid periodic fading previously mentioned and illustrated in Figures 9 and 17 should have some significance, since there is a considerable degree of regularity in its appearance, at least in reception from the two stations upon which observations have been most consistently made. During the cooperative investigation of fading: conducted by the Bureau of Standards in 1925 this same phenomenon was noted, ${ }^{9}$ though less consistently, in observations made at several different localities on transmissions from WGY (790 kc), Schenectady, $\mathrm{N}$. Y. In the earlier case the phenomenon occurred for a few days at the spring equinox and was not found again. The newer cases were first noted at the same season but have continued for several months with very few exceptions whenever records were made. On the WJZ records this type of fading starts from 15 to 30 minutes after the Washington sunset and lasts in the vicinity of a half hour. On the WBAL records, which were made much less frequently, the start is likely to be practically at sunset, though there is some variation both ways.

The explanation of this phenomenon suggested in the earlier paper as an interference effect between ground and reflected rays is now proven unsatisfactory by the fact that this periodic fading is present even when the ground ray is absent. As previously stated, the fact that such fading is out of phase on records made with coils at maximum and minimum positions, respectively, makes any interference explanation difficult and suggests a relatively weak indirect ray with rapid rotation of its plane of polarization. No explanation is given at this time for the rotation itself.

8 Reference 3, p. 313, and fig. 10.

- Reference 2, pp. 437-439 and fig. 5, opposite p. 427. 


\section{CONCLUSIONS}

1. Considerable fading is caused by the fact that the indirect ray from a radio broadcasting station undergoes variations of intensity before arriving at the receiving antenna. This is proved by the fact that at times similar intensity changes occur simultaneously in coil antennas at maximum and minimum positions as well as in barrage antenna and coil at minimum.

2. Much evidence of fading caused by interference between ground and indirect rays is found in records of reception from transmitting stations sufficiently near to produce a ground wave at the receiving point. The nighttime intensity is often less than the daytime intensity which is due to the constant ground ray. The out-of-phase indirect ray often neutralizes the effect of the ground ray partially, and sometimes completely, even for short-transmission paths.

3. Direction shifts are apparently necessary to the explanation of phase displacements of less than $180^{\circ}$ between otherwise similar records made simultaneously (1) with coil antenna at maximum and with vertical single-wire antenna, (2) with coil antenna at maximum and with barrage antenna, (3) with barrage and vertical single-wire antennas, (4) with coil antennas at maximum and minimum positions, and (5) with barrage and with coil antenna at minimum.

4. No proof of fading caused by fluctuating height of the ionized layer is found, but evidence of refraction of the indirect ray from a rising layer is found in the fact that the signal intensity in the coil antenna at minimum position starts at zero in daylight and gradually increases during sunset.

5. Rotation of plane of polarization of radiation refracted from the upper atmosphere is shown to be the cause of much fading, particularly, though by no means wholly, during the sunset period. The $180^{\circ}$ out-of-phase relationship between records made simultaneously with coil antennas in maximum and minimum positions seems to demand this explanation. The cause of such rotation is not explained.

6. Reflections arriving by multiple paths are evidenced in records showing a periodic type of fading superposed on the main intensity variations and yet $180^{\circ}$ out of phase on simultaneous records made with coil antennas in maximum and minimum positions. This fact makes the calculation of the effective height of the ionized layer by means of the sine of the angle of incidence, as determined by the method of Appleton and Ratcliffe, ${ }^{10}$ somewhat doubtful, especially when measuring on other than distant stations.

7. The rapid periodic type of fading noted in a previous paper as occurring in signals from one station during the sunset period has been found to be quite common in the reception from two other stations. 
The newer data point toward rotation of plane of polarization rather than interference as the cause.

As stated in the introduction, this investigation is far from complete. It is planned to make similar measurements over a much longer period of time to observe possible seasonal effects; to make simultaneous measurements by more than two receiving systems over a wider range of frequencies; to compare results of transmissions perpendicular to the earth's magnetic field with those parallel to it; to make direction and oscillographic observations simultaneously with the fading records; to make more quantitative measurements of these observations and compare calculated heights of the refracting layer, as determined by the angle of incidence method, on transmissions involving a wide range of distances, in order to note whether there is greater consistency in measurements on the more distant stations.

\section{ACKNOWLEDGMENTS}

Special acknowledgment is made of the assistance of H. S. Shaw, of Exeter, N. H., whose contribution of the salary of a research associate made possible the carrying on of this work at the Bureau of Standards.

Acknowledgment is also made of the assistance of S. A. Buckingham, R. P. Battle, and T. R. Gilliland in preparing apparatus and making the large number of records required in this work.

\section{APPENDIX}

\section{SUMMARY OF CONCLUSIONS OF PREVIOUS INVESTIGATIONS}

Both Pickard, in his 1923 investigation, ${ }^{11}$ and Appleton and Ratcliffe in their recent work ${ }^{12}$ have given the chief credit for fading to intensity changes undergone in the path of the indirect ray. Pickard ascribes these changes to varying absorption and suggests a possible explanation, while Appleton and Ratcliffe find reason to suspect that interferences between rays traveling by multiple paths are the cause. These authors agree also in crediting interference between ground and indirect rays with a minor rôle in causing fading and practically eliminate direction shifts as a factor. Appleton and Barnett, ${ }^{13}$ as well as Smith-Rose and Barfield, ${ }^{14}$ had previously satisfied themselves that apparent direction shifts were due chiefly to elliptical polarization of the atmospheric ray and very little, if at all, to actual changes in lateral angle with the great circle plane through transmitting and receiving points. Pickard did not discuss changing angle of incidence nor rotation of the plane of polarization of the down-coming ray in

11 Reference 1, pp. 152-158.

12 Reference 3, pp. 314-315.

${ }^{13}$ E. V. Appleton and M. A. Barnett, On Some Direct Evidence for Downward Atmospheric Reflection of Electric Rays, Proc. Royal Soc. A, 109, pp. 621-641; 1925. See especially pp. 623 and 635-636.

14 R. L. Smith-Rose and R. H. Barfield, The Cause and Elimination of Night Errors in Radio-Direction Finding, J. Inst. of Elect. Engrs., 64, pp. 831-837; 1926. 
connection with his fading data. Appleton and Ratcliffe were unable to detect any evidence that these factors were affecting fading, ${ }^{15}$ in spite of the fact that rotation of the plane of polarization of the incident ray had been accepted as explanation for apparent direction shifts. The Bureau of Standards report ${ }^{16}$ on cooperative measurements in 1925, while accepting varying absorption as the more general cause of fading, gave considerable weight to interference effects so far as fading of nearer stations was concerned, but no data bearing upon the other factors were then secured.

While the present paper deals only with observations in the broadcast range of frequencies and with limited methods of analysis, it is well to have in mind the conclusions reached by investigations at other frequencies and by other methods. Working particularly with frequencies above $3,000 \mathrm{kc}$ Breit and Tuve ${ }^{17}$ found fading occurring independently of interference between ground and indirect rays and suggested multiple reflections and flickering effect due to wavy character of surface of the ionized region. Heising, ${ }^{18}$ as well as Taylor and Young, ${ }^{19}$ using an oscillographic method similar to that of Breit and Tuve found proof of a multiplicity of paths of different lengths. Heising found these regularly accompanied by fading and simultaneous with an apparent rising of the ionized layer. Rukop ${ }^{20}$ in working with transmission of pictures at high frequency had earlier secured evidences of multiple paths which were accompanied by fading which, however, was not necessarily associated with the multiplicity of paths. The path length changed at times as rapidly as $50 \mathrm{~km}$ a second, giving the Doppler effect of a change in frequency at the receiving system. From theoretical considerations Pedersen ${ }^{21}$ concluded that there should be an infinite number of downcoming rays at a given point on the earth's surface.

Fading produced by interference was found by Bown, Martin, and Potter ${ }^{22}$ in an oscillographic study of the distortion in broadcast reception within a given area, and oscillographic observations by Friis ${ }^{23}$ of reception on 18.75 megacycles proved consistent with the belief that fading is mainly caused by wave interference.

15 Reference 3, pp. 312-315.

${ }_{16}$ Reference 2, pp. 447-448.

17 G. Breit and M. A. Tuve, A Test of the Existence of the Conducting Layer, Phys. Rev., 28, pp. 554575, September, 1926 . See especially p. 564.

18 R. A. Heising, Experiments and Observations Concerning the Ionized Regions of the Atmosphere, Proc. Inst. Radio Engrs. 16, pp. 75-99; January, 1928.

${ }^{19}$ A. Hoyt Taylor and L. C. Young. Studies of High-Frequency Radio-Wave Propagation, Proc. Inst.

Radio Engrs., 16, pp. 561-578; May, 1928.

${ }^{20}$ Von H. Rukop, Die Bildtelegraphie als Untersuchungsmethode für die Ausbreitung der kurzen wellen, Elektrische Nachrichten-technik, 3, pp. 316-318, 1926.

${ }_{21}$ P. O. Pedersen, The Propagation of Radio Waves Along the Surface of the Earth and in the Atmosphere, p. 230. Copenhagen, 1927.

${ }^{22}$ R. Bown, De L. K. Martin, and R. K. Potter, Some Studies in Radio Broadcast Transmission, Proc. Inst. Radio Engrs., 14, pp. 57-131; February, 1926.

${ }^{23}$ H. T. Friis, Oscillographic Observations on the Direction of Propagation and Fading of Short Waves, Proc. Inst, Radio Engrs., 16, pp, 658-665; May, 1928. 
Very good evidence of real direction shifts was found by Friis ${ }^{23}$ using a cathode-ray oscillograph to observe the phase difference of signal waves as received by antenna systems located a fraction of a wave length apart.

Changes in angle of incidence were found by Friis ${ }^{23}$ to be much more frequent and of greater magnitude than direction shifts of the received signals. Heising, by his observations of signal retardation, had previously computed changes in angle of incidence sufficient to indicate rapid variations in the height of the ionized layer, sometimes as great as $72 \mathrm{~km}$ (45 miles) in two minutes.

Rotation of the plane of polarization of the indirect ray was suggested from theoretical considerations of the earth's magnetic field and varying ionization in the upper atmosphere by Appleton and Barnett ${ }^{24}$ and by Nichols and Schelleng, ${ }^{25}$ who indicated that this phenomenon might be expected in transmissions largely along the line of a magnetic meridian. Evidences of abnormal polarization causing apparent direction shifts were pointed out by Eckersley, ${ }^{26}$ and Pickard ${ }^{27}$ found by many observations made on high frequencies with an analyzer that the plane of polarization under certain conditions rotated $90^{\circ}$ in transit. Alexanderson's investigation, ${ }^{28}$ also on the high frequencies, seemed to indicate a continuous rotation of the plane of polarization with alternate points of plane and circular polarization along the transmission path. Hollingworth ${ }^{29}$ recently made measurements to determine the state of polarization of the indirect ray which produces apparent progressive direction shifts during the sunset period, and on the basis of several assumptions calculated that the plane of polarization of an indirect ray received from a selected transmission of $21 \mathrm{kc}$ rotated $90^{\circ}$ during sunset and remained thus abnormally polarized during the night. Eckersley, ${ }^{30}$ working with the high frequencies, made simultaneous fading records with horizontal and vertical antennas and reports that under some conditions the fading changes in the two records were so nearly opposite in phase as to indicate rotation of the plane of polarization of the indirect ray.

Washington, December 8, 1928.

${ }^{24}$ E. V. Appleton and M. A. F. Barnett, Wireless-wave propagation, Electrician, 94, p. 398; Apr. 3, 1925.

25 H. W. Nichols and J. C. Schelleng, The Propagation of Radio Waves Over the Earth, Nature, 115, p. 334; Mar. 7, 1925; also Bell System Tech. J., 4, pp. 215-234; April, 1925.

${ }_{28}$ T. L. Eckersley, The Effect of the Heaviside Layer on the Apparent Direction of Electromagnetic Waves, Radio Review, 2, pp. 60-65, and 231-248; February and May, 1921.

${ }^{27}$ G. W. Pickard, The Polarization of Radio Waves, Proc. Inst. Radio Engrs., 14, pp. 205-212; April, 1926.

${ }^{28}$ E. F. W. Alexanderson, Polarization of Radio Waves, J. Am. Inst. Elect. Engrs,. 45, pp. 636-640; July, 1926.

${ }_{29}$ J. Hollingworth, The Polarization of Radio Waves, Proc. Royal Soc., A, 119, pp. 444-464; 1928.

${ }^{30}$ See footnote 6, p. 1066 . 